\title{
Disparities in colorectal cancer early detection and prevention among sub-populations in Israel: Patient activation score according to mother tongue, gender, and age
}

\author{
Sarit Balanson ${ }^{1 *}$, Jesse Lachter ${ }^{2,3}$, Hana Lupo-Gralnik ${ }^{3}$ and Yakov Fogelman ${ }^{3}$ \\ ${ }^{1}$ Technion Israel Institute of Technology, Haifa, Israel \\ ${ }^{2}$ Department of Gastroenterology, Rambam Healthcare Center, Technion Israel Institute of Technology, Haifa, Israel \\ ${ }^{3}$ United (Meuhedet) Healthcare Service, Israel
}

\begin{abstract}
Objective: To determine concrete disparities in CRC prevention among Israelis of varying mother tongues while considering the role of patient activation.

Methods: This study took place in Haifa, Israel from August 2015-February 2017. Questionnaires were distributed at Rambam Healthcare Campus and two health maintenance organization (HMO) clinics. Participants were compared by variables including: mother tongue, CRC prevention, age, gender, and PAM Score.

Results: Analysis of 221 participants found PAM scores differ significantly by gender and, in some cases, mother tongue. Older and more activated patients were more likely to perform CRC screening (0.02 and 0.49 respectively).

Discussion: Differences in PAM Score and patient activation among sub-populations were observed. Significant differences exist among stratified populations with respect to CRC screening; overall, more activated patients performed CRC screening. PAM Scores are currently not included as part of the assessment of health inequalities by large health organization, however, target populations may benefit from this evaluation via cost-reductions and enhanced medical care.
\end{abstract}

Conclusion: Significant differences were observed among sub-populations whereby more activated patients were more likely to perform CRC screening.

\section{Introduction}

Disparities in healthcare are ubiquitous. Often, these disparities tend to plague individuals of low socioeconomic and/or immigrant status [1]. As immigration throughout the world continues to increase, the Organization for Economic Co-operation and Development (OECD) reported a 65\% increase in the global North since the 1990s, identifying disparities and generating strategies to ameliorate gaps is becoming increasingly important [2,3]. Israel, like many other countries, has experienced a large influx of immigrants since the 1990s, specifically from the former USSR $(1,013,221)$, Ethiopia $(75,196)$, and USA $(38,858)$ [3]. These populations bring with them diverse cultural attitudes relating to healthcare that inform their experience of healthcare in their new country. To better incorporate new citizens, close existing healthcare gaps, and prevent new gaps from forming, policy makers must consider patient activation, patient satisfaction, and patient experience through the lens of the immigrant. In articles by Serper et al. [4] and Hibbard et al. [5] patient activation is defined as "an individual's knowledge, skill and confidence for managing his/her own health care" where the patient can "participate as an active member of the care team". This activation depends on the patient's health literacy, the ability of the patient to understand health information, and services available to make suitable health decisions [4].

Understanding the capacity of immigrant populations to comprehend and solve health problems on their own better defines the roles of the physician and the patient. While patient activation is subjective, Hibbard et al has generated and validated a Patient
Activation Measurement (PAM) Score that can be assessed with a 10-question questionnaire, which has been licensed for use in this this study. Hibbard's PAM Score is considered the gold standard for evaluating patient activation. The survey has been validated and used in over 200 peer-reviewed published studies and is used in over 16 countries [6-8]. Per the license from Insignia Health, the PAM Score tool "assesses a consumer's knowledge, skills and confidence for self-management" [9]. Responses to the questions are entered a table and results in a figure that places the patient into one of four categories indicating a level of patient activation. The level one patient is disengaged and overwhelmed while a level two patient is becoming aware, but still struggling. A level three patient is acting while a level four patient is maintaining healthy behaviors and pushing further to improve their care. Over time a patient's score can change as they become activated. Ultimately, the goal of PAM is to be employed as a tool to initiate discussion and encourage improved activation [9].

Patient satisfaction and experience are even more difficult to define and quantify due to subjectivity. Much of the relevant literature is unclear about the role of patient experience and how expectations of

*Correspondence to: Sarit Balanson, Technion Israel Institute of Technology, Haifa, Israel, Tel: +972-54-431-2480; E-mail: sbalanso@gmail.com

Key words: health inequality, patient activation, colorectal cancer screening

Received: August 07, 2018; Accepted: August 20, 2018; Published: August 24 2018 
Balanson S (2018) Disparities in colorectal cancer early detection and prevention among sub-populations in Israel: Patient activation score according to mother tongue, gender, and age

experience inform satisfaction; however, expectations, which include needs, requests, and desires, play a major role in patient satisfaction $[10,11]$. Other studies like one by Ahmed et al show that these variables are unrelated and depend on individual personality and ability to deal with change [11]. More generally, however, and more relevant to this study, is the idea that differences in patient experience and satisfaction may reflect differences in expectations related to the patients' background [12]. This has been observed in varied ethnic populations [10].

In Israel, studies on healthcare disparities often focus on the difference between Arab and Jewish communities, not other subpopulations in Israel. For example, Baron-Epel et al. [13] note that Arab and Jewish populations use the National Health Insurance differently, Arabs were more likely to visit family physicians while Jews were more likely to see specialists. Further, the study notes that socioeconomic status could not explain this difference. The reason for this difference is complex with no single answer [14]. Cultural differences and patient activation may play a big role. Besides academic research teams, various organizations have been investigating health inequalities in Israel. The Joint Distribution Committee (JDC) is one of those organizations and, in 2006, the Smokler Center for Health Policy Research published a report entitled Reducing Health Inequality in Israel: Towards a National Policy and Action Program. Importantly, the study notes that health inequality is not just a social issue. Indeed, there are far-reaching consequences including economic losses for days lost from work as well as increased cost of care for sicker patients who are not regularly cared for [14].

The above-noted disparities can be difficult to concretize, as they are often subjective with confounding factors. Therefore, our study includes a quantitative measurement. We chose colorectal cancer (CRC) screening via either colonoscopy or fecal occult blood test (FOBT), or both. The choices available in Israel for CRC screening consider FOBT as the preferred test. Colonoscopy is widely used for screening but is not in the official health basket of services provided by the four government-regulated health maintenance organizations (HMOs), which provide universal coverage for citizens. The rates of screening tests like CRC screening and other preventative tests in general are lowest among populations of immigrants and those with low socio-economic standing, as was found in a population-based study by Borkhoff et al. [1] across Ontario, Canada. Therefore, CRC screening was posited to be a good surrogate marker of patient activation and participation in care, especially among immigrant populations.

Additionally, the research was conducted in affiliation with the Department of Gastroenterology at Rambam Healthcare Campus and two HMOs. One goal of this study was to assess and expand the ideas presented by Baron-Epel et al. [13] and Rosen B [14] to include important sub-populations (defined via mother tongue: English, Arabic, Russian, Amharic, and Hebrew) that have varied cultural backgrounds. Further, and more specifically, the aim of this study was to determine the content of any disparities with respect to patient activation and CRC screening (via colonoscopy or FOBT) and understand how these differences relate to mother tongue and other demographics. Additionally, we aimed to explore any noted disparities that might be addressed. Due to the inconcrete nature of health disparities we have decided to assess the more general PAM score as associated with the concrete measurement of the rate of CRC screening.

\section{Materials and methods}

The questionnaire used was designed by Dr. Sarit Balanson. Sarit received assistance from Ronit Leiba, Rambam statistician, who provided guidance and examples questionnaires that had been distributed previously at Rambam Hospital. Further, the official PAM Score questionnaire was licensed from Insignia Health [5,6]. The PAM Score was available only in English so, with the help of Dalia Katz and Prof. Jesse Lachter, the questions were translated into Hebrew.

A proposal was drafted and submitted for approval to Helsinki. After approval (\#0308-15-RMB), questionnaires were distributed at Rambam (Internal Medicine D, outpatient clinics, Gastroenterology Department) and two-family medicine clinics (Leumit, Meuhedet). Questionnaires were administered by Dr. Sarit Balanson, Prof. Jesse Lachter, Dr. Hana Lupo, Dr. Yaakov Fogelman, and nurse/research assistant Dora Hagalili and completed by patients.

Informed consent was obtained from all individual participants included in the study. A total of 304 individuals participated and the preliminary results included all participants. However, upon assessment, participants below age 50 were excluded from most of the analysis as the recommendation for CRC screening per the United States Preventative Services Task Force (USPSTF) is 50 years and above [15]. Therefore, most results include individuals over 50 years, totaling 221 patients $(n=221)$. Additionally, 72 patients $(n=72)$ under 50 were included in a comparison analysis. Participants were frequently unable to complete questionnaires independently resulting in many cases where the questions were dictated to the patient and the interviewer marked down the responses. Those that did complete the questionnaires independently often omitted some answers therefore some categories lack all responses. Paper questionnaires were gathered by Dr. Balanson and entered into an Excel table, generating an electronic database, which was used to perform statistical analysis. Analyses were performed using the SPSS program version 21. Descriptive statistics were used for mean, median, standard deviation, percentiles, and ranges. T-test and Pearson correlation were used for differences in quantitative parameters. Fisher exact tests and Pearson Chi-Squared were used for differences in categorical parameters. A $p$-value $<0.05$ was the cut-off for values considered to be significant.

\section{Results}

A total of 221 questionnaires from participants over 50 were used in the following analysis. At the end of the results section there is a table of comparison analysis that includes the 72 participants under 50. Data was gathered including details about demographics, patient activation, and CRC screening. Characteristics of the population are both noted here and summarized in table 1 . The study population included more women than men (118 [55\%] versus 97 [45\%]) and more Hebrew speakers than those of other mother tongues (122 [58\%] versus 89 [42\%]). The population included individuals with varied mother tongues: Hebrew, Arabic, Amharit, Russian, and English. Further, the population was composed of both immigrants and those born in Israel. Lastly, age groups of the population were also recorded.

Not all questionnaires were completed and often a few spaces were left blank. In such cases, the questionnaires were still included in analysis with the parts left blank excluded from calculations. Mean PAM Scores were calculated with most participants having a score of 2 or 3 ( $25 \%$ and $40 \%$ of the study population respectively). Mean PAM Scores were then analyzed by various patient characteristics including mother tongue, gender, and age group. By mother tongue, the mean PAM scores were 62.6 for Hebrew speakers, 57.4 for Arabic speakers, and 50.5 for Russian speakers. When comparing native Russian speakers to those with Hebrew as a mother tongue, the difference in PAM Score was significant with $p<0.0001$. Participants' whose native tongue was 
Balanson S (2018) Disparities in colorectal cancer early detection and prevention among sub-populations in Israel: Patient activation score according to mother tongue, gender, and age

Table 1. Study Population Characteristics. Note the differences in gender, mother tongue, number of years living in Israel, and age. All values are listed in as both a count and percentage

\begin{tabular}{|c|c|}
\hline Category & Count (Percentage) \\
\hline Gender & $118(55 \%)$ \\
\hline Memale & $97(45 \%)$ \\
\hline & Mother Tongue \\
\hline Hebrew & $122(58 \%)$ \\
\hline Arabic & $43(20 \%)$ \\
\hline Amharit & $5(2 \%)$ \\
\hline Russian & $31(15 \%)$ \\
\hline English & $10(5 \%)$ \\
\hline & Number of Years in Israel \\
\hline Born & $118(55 \%)$ \\
\hline More than 20 & $87(40 \%)$ \\
\hline 11 to 20 & $8(3.5 \%)$ \\
\hline 5 to 10 & $1(0.5 \%)$ \\
\hline Less than 5 & $2(1 \% \%)$ \\
\hline Age & $89(40 \%)$ \\
\hline $50-60$ & $132(60 \%)$ \\
\hline$>60$ &
\end{tabular}

Amharic, or English were so few that they have been excluded from this analysis, 5 and 10 individuals respectively. By gender, females were more activated than males with an average PAM score of 62.3 versus 57.1. This result was significant with a $p=0.016$. Finally, by age, the there was no significant difference between the PAM score of those 51-60 or $>60$ with average values of 60.9 and 59.1 respectively, $p=$ 0.38. Results of mean PAM score by age, gender, and mother tongue are summarized in figure 1.

CRC screening was calculated by responding 'yes' to having undergone a colonoscopy or FOBT. Of the 221 participants, 164 had completed either a colonoscopy or FOBT. Of those 164, there were no significant differences between groups by gender or mother tongue. However, patients over 60 were more likely to have performed FOBT or colonoscopy when compared to those 50-60, $p=0.02$ (Table 2). Moreover, those who completed CRC screening had significantly higher mean PAM 10 Scores than those who did not (61.06 versus $56.12, p=0.49$ ).

Finally, a comparison of patients over age 50 and those under age 50 with respect to $\mathrm{CRC}$ prevention screening was performed. Older patients significantly performed FOBT more frequently than younger, $94(48 \%)$ as compared to $17(25 \%), p=0.001$. However, the difference in colonoscopy was not significant, $136(62 \%)$ in the older population compared to $35(49 \%)$ in the younger population $(p=0.053)$. Table 3 displays this data as well as other demographic information about the older and younger populations. There is no significant difference with respect to gender or mother tongue among the different age groups.

\section{Discussion}

The population in this study contained more women than men as well as a disproportionate amount of native Hebrew speakers and individuals born in the Israel, with fewer immigrants. Our study attempted to assess for differences in patient activation and CRC screening among varied sub-populations that were defined by gender, age, and mother tongue. We found that women are significantly more activated than men with overall higher PAM Scores ( $p$-value 0.016). Further, native Hebrew speakers were more activated than native Russian speakers ( $p$-value $<0.0001)$ and Russian speakers were more activated than English and Amharic speakers, but there was no significant difference between native Hebrew and native Arabic speakers. The questionnaire asked about years working and job type to better understand the level of education and lifestyle of the subjects. However, due to insufficient responses, the data was not included in the analysis. There were no significant differences in PAM Score with respect to age group. Regarding CRC screening, measured by completion of a colonoscopy or FOBT, there were no significant results with respect to gender and mother tongue suggesting that neither group was more likely to complete CRC screening. However, there were significant results with respect to age with older population $(>60)$ being more likely to complete a colonoscopy or FOBT versus a younger population $(50-60)(80.9 \%$ versus $66.7 \% p=0.024)$. Further, our data

Table 2. CRC Screening by gender, mother tongue, and age. Tabulated information regarding FOBT or colonoscopy with respect to population details can be seen below. The details include population details about gender, mother tongue and age. Results are recorded in count and percentages and p-values are noted

\begin{tabular}{|c|c|}
\hline \multicolumn{2}{|c|}{ CRC screening (FOBT or colonoscopy) } \\
\hline Category & Count (Percentage) \\
\hline Gender & $P=0.63$ \\
\hline Male & $74(77.1 \%)$ \\
\hline Female & $86(73.5 \%)$ \\
\hline Mother Tongue & $P=0.61$ \\
\hline Hebrew & $96(78.7 \%)$ \\
\hline Arabic & $29(69.0 \%)$ \\
\hline Russian & $22(73.3 \%)$ \\
\hline Amharit \& English & $10(71.4 \%)$ \\
\hline Age & $P=0.02$ \\
\hline $50-60$ & $58(66.5 \%)$ \\
\hline$>60$ & $106(80.9 \%)$ \\
\hline
\end{tabular}

Table 3. Demographic and CRC screening data comparing participants under 50 years old to those over 50 years old

\begin{tabular}{|c|c|c|}
\hline & Under Age $50(\mathrm{n}=72)$ & Over Age $50(n=221)$ \\
\hline \multicolumn{3}{|c|}{ Gender $(p=0.67)$} \\
\hline Male & $33(49 \%)$ & $97(45 \%)$ \\
\hline Female & $35(51 \%)$ & $118(55 \%)$ \\
\hline \multicolumn{3}{|c|}{ Mother Tongue $(p=\mathbf{0 . 5 0})$} \\
\hline Hebrew & $39(55 \%)$ & $122(58 \%)$ \\
\hline Arabic & $20(28 \%)$ & $43(20 \%)$ \\
\hline Russian & $9(13 \%)$ & $31(15 \%)$ \\
\hline Amharit and English & $3(4 \%)$ & $15(7 \%)$ \\
\hline FOBT Performed $(p=0.001)$ & $17(25 \%)$ & $94(48 \%)$ \\
\hline Colonoscopy Performed $(p=0.053)$ & $35(49 \%)$ & $136(62 \%)$ \\
\hline
\end{tabular}

\section{Mean PAM Score by Age, Gender, and Mother Tongue}

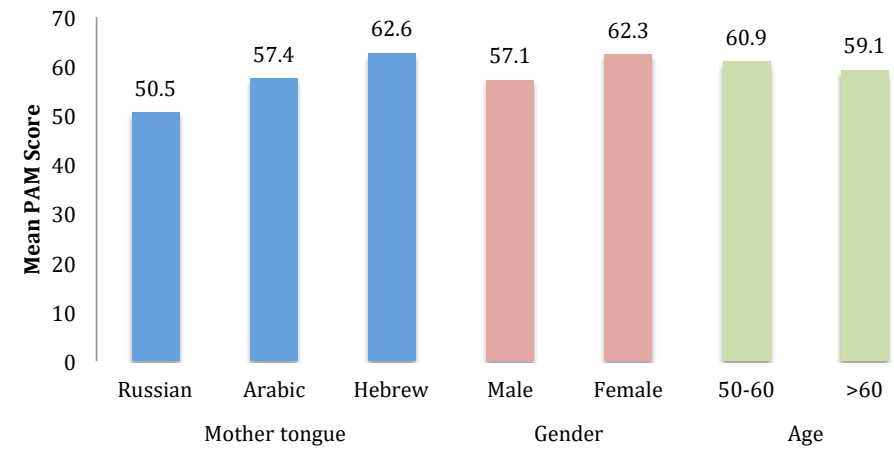

Figure 1. The figure demonstrates the mean PAM score as it relates to mother tongue, gender, and age. Mean values are shown side-by-side to allow for easier comparison 
Balanson S (2018) Disparities in colorectal cancer early detection and prevention among sub-populations in Israel: Patient activation score according to mother tongue, gender, and age

supported the hypothesis that more activated patients are more likely to complete CRC screening tests $(p=0.49)$.

\section{PAM score and CRC screening}

An important finding of this study was the differences among sub-populations with respect to patient activation. Patient activation requires a detailed understanding to interpret its meaning. Patient activation is important because more activated patients are more involved and in charge of their healthcare-this means a patient is a more aware or 'active' participant in their care. Moreover, research has demonstrated that "levels of patient activation are related to most health behaviors, many clinical outcomes, health care costs and patient experiences" as well as a predictor of health outcomes [16-18]. Interpreting patient activation depends on which category subjects fall into based on their responses to the questionnaire. There are four categories. Per the creators of PAM Score, a patient with an activation score of one does not even understand that they have role in their healthcare while those with a score of two "lack basic knowledge about their condition, treatment options, and/or self-care" [9]. Level three patients understand basic concepts related to their healthcare, have participated in their care, and have some confidence in these actions. Finally, those whose activation is level four, the highest level, are knowledgeable and feel in control of their health but still need their physician's support [9]. See tables for more details. PAM Score is a measurable way to understand health inequality among different groups within the population. Health inequality is a very broad topic with many contributing factors, which is not within the scope of this discussion to review. Many studies, such as the recent Katikireddi et al. [19] longitudinal study, focus on socioeconomic and lifestyle factors (smoking, alcohol, diet, body mass index [BMI], and physical activity) that contribute to multimorbidity. These factors are alterable independently, but also as a facet of patient activation. Moreover, in our heterogeneous society, health inequality is pervasive and does not necessarily sit along the well-carved socioeconomic or demographic lines. The PAM Score uniquely ignores socioeconomic and demographic factors [17]. One way to combat health inequality is to create more activated patients once we know their base activation score. Physicians play an important role in the enhancement of patient activation. Various think tanks have explored the concept of health inequality in Israeli society. More specifically, the JDC in their publication about health inequalities in Israel note that to truly fix these disparities there needs to be a change in how we educate our medical students and physicians using "training frameworks to prepare professionals to address the health effects of socioeconomic and cultural inequality" [14]. The JDC also suggests that specific sub-populations need to be targeted based on their needs as differences in socioeconomic and cultural backgrounds greatly influence health. Perhaps including PAM Scores by population can help better target therapies and interventions. Thus, one outcome of the current study is to focus on empowering native speakers of Russian, a large group in Israel, to trend towards selfadvocacy in their health maintenance. Previous research studies have analyzed the PAM Score and its relationship to other clinical factors. Empirical studies have demonstrated that patients who are more activated are also more likely to take preventative health measures like participating in screening tests, regular check-ups, and immunizations [16]. Studies by Greene and Hibbard et al. [16] and Tabrizi et al. [17] have shown that more activated patients are more likely to have a healthy diet and regularly exercise along with other health-conscious behaviors [20,21]. Additionally, in management of chronic diseases, more activated patients have better and more successful management [20-23]. A literature survey shows no studies that assess how PAM
Scores relate to specific quantifiable screening tests like colonoscopy/ FOBT, mammography, or PAP smears.

However, there is abundant literature exploring other factors that contribute to disparities in quantifiable screening tests among sub-populations. For example, in CRC screening, studies note that individuals of lower socioeconomic status are less likely to screen and, if they have CRC, are more likely to be diagnosed at a late stage $[24,25]$. Late-stage diagnosis was also associated with non-white ethnicity and socially deprived status. The role of physicians here is also important, as the family doctor needs to proactively recommend the screening tests to the patient. Indeed, a study by Folassade et al. [26] notes that racial minorities are less likely to receive CRC prevention recommendations from their physician. Physicians might also consider providing public lectures on CRC screening and prevention, as they have proved effective among Israelis as seen in an article by Lachter et al. [27]. These factors in addition to the level of patient activation contribute to health inequalities among sub-populations throughout the world and should be considered when interpreting the data.

\section{Conclusion}

The results from our study indicate that there are significant differences among sub- populations with respect to patient activation and CRC screening. Further, the results demonstrate a correlation between a patients' level of activation and their participation in screening tests. This topic is important to consider in achieving a healthier population in Israel. This study served as a pilot study to allow for exploration of the analysis and methods. Moving forward, we plan to expand the study to only include those over the age of 50 . We will also try to only use patients from family medicine clinics to control for confounders. Many organizations including the Ministry of Health, OECD, the HMO Clalit, and the JDC have drafted potential plans to reduce health inequalities [14,29-31]. However, none of these plans include augmenting patient activation. Our hope is that by employing PAM Scoring we can identify subjects needing encouragement and empowerment become more activated patients, and concomitantly promoting a cultural sensitivity focus among healthcare providers. The ultimate goals include enhancing CRC prevention as well as other healthy behaviors. This research provides another target and tool to improve our population's health.

\section{References}

1. Borkhoff CM, Saskin, R, Rabeneck L, Baxter NN, Liu Y, et al. (2013) Disparities in receipt of screening tests for cancer, diabetes, and high cholesterol in Ontario, Canada: a population-based study using area-based methods. Can J Public Health 104: e284e290. [Crossref]

2. World Migration in Figures (2013) Available at: https://www.oecd.org/els/mig/WorldMigration-in-Figures.pdf.

3. Statistical Abstract of Israel. (2014) Available at: http://cbs.gov.il/reader/shnaton/ shnatone_new.htm?CYear $=2014 \& \mathrm{Vol}=65 \& \mathrm{CSubject}=4$.

4. Serper M, Gawron AJ, Smith SG, Pandit AA, Dahlke AR, et al. (2014) Patient factors that affect quality of colonoscopy preparation. Clin Gastroenterol Hepatol 12: 451-457. [Crossref]

5. Hibbard JH, Stockard J, Mahoney ER, Tuslet M (2004) Development of the Patient Activation Measure (PAM): Conceptualizing and Measuring Activation in Patients and Consumers. Health Serv Res 39: 1005-1026. [Crossref]

6. Maindal HT, Sokolowski I, Vedsted P (2009) Translation, adaptation and validation of the American short form Patient Activation Measure (PAM13) in a Danish version. BMC Public Health 9: 209. [Crossref]

7. Brenk-Franz K, Hibbard JH, Hermann WJ, Freund T, Szecsenyi J, et al. (2013) Validation of the German version of the patient activation measure (PAM13-D) in an international multicenter study of primary care patients. PLoS One 8: e74786. [Crossref] 
Balanson S (2018) Disparities in colorectal cancer early detection and prevention among sub-populations in Israel: Patient activation score according to mother tongue, gender, and age

8. Rademakers J, Nijman J, van der Hoek L, Heijmans M, Rijken M (2012) Measuring patient activation in The Netherlands: translation and validation of the American short form Patient Activation Measure (PAM13). BMC Public Health 12: 577. [Crossref]

9. Patient activation measure license materials (2014) Insignia Health, LLC. Available at: http://solihulltogether.co.uk/images/Patient_Activation/UK_PAM_13.pdf

10. Ahmed F, Burt J, Roland M (2014) Measuring patient experience: concepts and methods. Patient 7: 235-241. [Crossref]

11. Reiff M, Zakut H, Weingarten MA (1999) Illness and treatment perceptions of Ethiopian immigrants and their doctors in Israel. Am J Public Health 89: 1814-1818. [Crossref]

12. Baider L, Ever-Hadani P, De-Nour AK (1995) The impact of culture on perceptions of patient-physician satisfaction. Isr J Med Sci 31: 179-185. [Crossref]

13. Baron-Epel O, Garty N, Green MS (2007) Inequalities in use of health services among Jews and Arabs in Israel. Health Serv Res 42: 1008-1019. [Crossref]

14. Rosen B (2006) Reducing Health Inequality and Health Inequity in Israel: Towards a National Policy and Action Program - Summary Report. Available at: https:// brookdale.jdc.org.il/en/publication/reducing-health-inequality-health-inequity-israeltowards-national-policy-action-program-summary-report/.

15. The Guide to Clinical Preventive Services (2014) Available at: https://www.ahrq gov/sites/default/files/wysiwyg/professionals/clinicians-providers/guidelinesrecommendations/guide/cpsguide.pdf.

16. Hibbard J, Gilburt H (2014) Supporting people to manage their health. An introduction to patient activation. Available at: https://www.kingsfund.org.uk/sites/default/files/ field/field_publication_file/supporting-people-manage-health-patient-activationmay14.pdf.

17. Wisbaum W (2015) Promoting better health and better care at lower cost: The Patien Activation Measure (PAM). Available at: http://s3-us-west-2.amazonaws.com/ insignia/Funcas.Promoting-Better-Health-and-Better-Care-at-Lower-Cost-with-PAM. pdf?mtime $=20151216140434$.

18. Skolasky RL, Green AF, Scharfstein D, Boult C, Reider L, et al. (2011) Psychometric Properties of the Patient Activation Measure among Multimorbid Older Adults. Health Serv Res 46: 457-478. [Crossref]

19. Katikireddi SV, Skivington K, Leyland AH, Hunt K, Mercer SW (2017) The contribution of risk factors to socioeconomic inequalities in multimorbidity across the lifecourse: a longitudinal analysis of the Twenty-07 cohort. BMC Medicine 15: 152. [Crossref]
20. Greene J, Hibbard JK (2012) Why does patient activation matter? An examination of the relationships between patient activation and health-related outcomes. $J$ Gen Intern Med 27: 520-526. [Crossref]

21. Tabrizi JS, Wilson AJ, O'Rourke PK (2010) Customer quality and type 2 diabetes from the patients' perspective: a cross-sectional study. J Res Health Sci 10: 69-76. [Crossref]

22. Ellins J, Coulter A (2005) How engaged are people in their health care? Findings of a national telephone survey. Available at: https://www.picker.org/wp-content/ uploads/2014/10/How-engaged-are-people-in-their-health-care-....pdf.

23. Hibbard JH, Cunningham PJ (2008) How engaged are consumers in their health and health care, and why does it matter? Res Brief 8: 1-9. [Crossref]

24. de Klerk CM, Gupta S, Dekker E, Essink-Bot ML, Expert Working Group, et al. (2017) Socioeconomic and ethnic inequities within organized colorectal cancer screening programmes worldwide. Gut 67: 679-687. [Crossref]

25. Askari A, Nachiappan S, Currie A, Lachford A, Stebbing J, Bottle A, et al. (2017) The relationship between ethnicity, social deprivation and late presentation of colorectal cancer. Cancer Epidemiol 47: 88-93. [Crossref]

26. Folassade P, Almario CV, Ponce, N, Speigel BM (2015) Racial Minorities Are More Likely Than Whites to Report Lack of Provider Recommendation for Colon Cancer Screening. Am J Gastroenter 110: 1388-1394. [Crossref]

27. Lachter J, Leska-Aharoni T, Warum D, Eliakim R (2008) Overcoming barriers to colorectal cancer screening tests. Isr Med Assoc J 10: 621-626. [Crossref]

28. Magnezi R, Glasser S (2014) Psychometric Properties of the Hebrew Translation of the Patient Activation Measure (PAM-13). PLoS One. 9: e113391. [Crossref]

29. Horev T, Averbuch E (2012) Coping with Health Inequalities: A roadmap for developing a national plan. The Israeli Experience. Available at: https://www.health. gov.il/PublicationsFiles/HealthInequalities_roadmap04042012.pdf.

30. OECD Reviews of Health Care Quality: Korea (2012) Available at: http://www.oecd org/publications/oecd-reviews-of-health-care-quality-korea-9789264173446-en.htm.

31. Balicer RD, Shadmi E, Lieberman N, Greenberg-Dotan S, Goldfracht G, et al. (2011) Reducing Health Disparities: Strategy Planning and Implementation in Israel's Largest Health Care Organization. Health Serv Res 46: 1281-1299. [Crossref]

Copyright: (C2018 Balanson S. This is an open-access article distributed under the terms of the Creative Commons Attribution License, which permits unrestricted use, distribution, and reproduction in any medium, provided the original author and source are credited. 